INTERNATIONAL DESIGN CONFERENCE - DESIGN 2018

https://doi.org/10.21278/idc.2018.0416

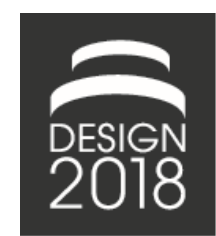

\title{
INSIGHTS ON HOW METACOGNITION INFLUENCES KNOWLEDGE APPLICATION IN PRODUCT DESIGN EDUCATION
}

\author{
Y. Zhang, E. Bohemia and J. McCardle
}

\begin{abstract}
An empirical study investigating creativity relevant factors that impact on knowledge application within the context of Product Design Education has been undertaken in the form of survey. The principal creativity relevant factor is identified as metacognition, which is related to creative thinking. Different kinds of knowledge applied in Product Design students' final year design projects (FYDPs) have been assembled and arranged into three categories. Possible ways of categorising knowledge according to the influences of metacognition are proposed that may inform design education practices.
\end{abstract}

Keywords: design creativity, design knowledge, design education, domain knowledge, tacit knowledge

\section{Introduction}

This research investigates how creativity relevant factors affect subject-relevant knowledge application within the context of product design education. The research is sourced from a widely observed phenomenon that students of Product Design in China depend highly on their tutors' suggestions for the progression of final year design projects (FYDPs). It has been observed that although students are often considered by tutors to have mastered a range of knowledge after four years' study to support and engage with major projects, they still differ largely in the creative performance of their FYDPs.

According to cross-cultural studies (Zhang et al., 2017), the western culture and tradition focuses heavily on fostering creative thinking skills amongst students during learning and knowledge acquisition. However, in the Chinese culture, the convention is to emphasise the accumulation of knowledge as a fundamental objective with the expectation of the spontaneous appearance of creative thinking abilities as the knowledge accrues. Knowledge is, therefore, considered to consist of two parts, (i) the physical accumulation of knowledge and (ii) the creative ability to critically analyse and apply knowledge learnt. It may be that western culture places higher value on creative ability. It may be that Chinese students grasp knowledge well but lack creative relevant abilities. These speculations may link to the creative thinking ability referred to by Cropley (2000), and which is defined as the ability of "thinking that is novel and that produces ideas that are of value" (Sternberg, 2003, p. 326). A problem has already been identified by the authors as the lack of creative thinking ability with some students regarding the application of relevant knowledge appropriately when conducting certain aspects of their FYDPs, and consequently failing to deliver solutions creatively (Zhang et al., 2017).

This study aims to explore the interactions between creativity and knowledge application as currently there is little insight towards how creativity affects knowledge application, and specifically in the utilisation of knowledge and skills within or across diverse domains. The research question centres on how the relevant factor of creativity impacts the way of applying various types of knowledge in FYDP. Correspondingly, 
we argue that it is essential to understand creativity from a cognitive view and understand it as a thinking process involving interactions between divergent thinking and convergent thinking, where,

"convergent thinking usually generates orthodoxy, whereas divergent thinking always generates variability." (Cropley, 2006, p. 392), and both types of thinking work together "that allows the generation of ideas that are both original and effective" (Kozbelt et al., 2010, p. 32).

Based on this perspective, this study examined one of the creativity-relevant factors - metacognition impacts on knowledge application within product design domain. The study described here has applied the quantitative post-positive methodology, wherein an online survey-based questionnaire was designed to collect data on two psychometric measurements. Specifically, (i) For measuring the frequency of using different kinds of subject-relevant knowledge in the design process of FYDP; and (ii) For evaluating students' creative thinking abilities via measuring their metacognition.

\section{Literature review}

\subsection{Creativity and knowledge}

\subsubsection{Knowledge and creativity from the view of domains}

Alexander et al. (1991) defines knowledge as "an individual's personal stock of information, skills, experiences, beliefs and memories" (p. 317). In this study, we treat knowledge as a tangible concept in relation to design, and include a range of principles, skills, and subject relevant information (Christiaans, 1992).

When it comes to the nature of creativity, two contrasting views of whether it is domain-general or domainspecific have been comprehensively discussed within two diverse lines of study. Congruent to the suggestions of the majority of researchers that people's knowledge base plays a significant role in this conceptual process (Lawson, 2006); this debate also largely influences the study of knowledge in creativity. Amabile's (1996) Componential Model of Creativity (CMC) implies that the study of knowledge in creativity theories; all relevant information and knowledge, both domain-general and domain-specific, should be taken into account when solving a problem (Figure 1). As Adams (2005, p. 5) further explains "it is proposed the best profile for creativity is the T-shaped mind, with a breadth of understanding across multiple disciplines and one or two areas of in-depth expertise". This means that the technical expertise of an individual develops when he pays complete attention to a specific subject and accumulates various experiences to a certain degree, and this technical expertise can be applied as the foundation or resource to achieve and facilitate creativity. On the other hand, it is also essential and significant that an individual continues to retain broader thought-processes and is encouraged to encompass various interests to thrive creativity.

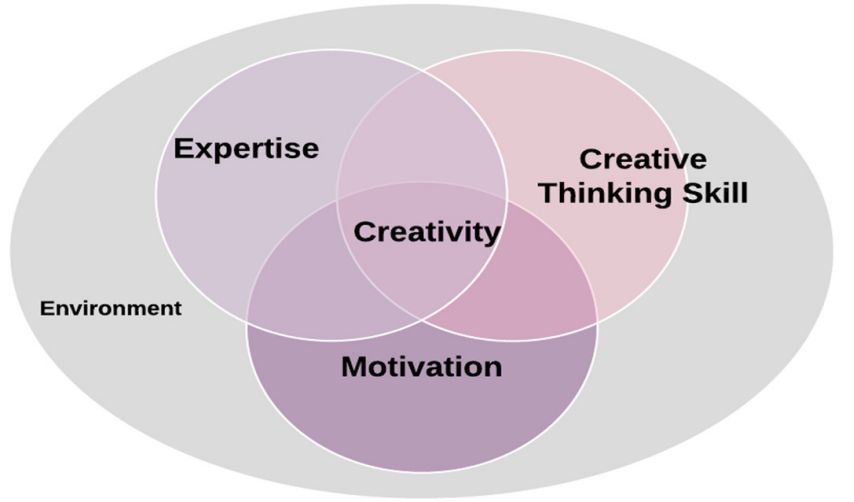

Figure 1. Componential Model of Creativity (Amabile, 1996)

Creativity theorists have a common belief with respect to the significance of domain-specific knowledge in creativity (Weisberg, 1999). Alternatively, general process knowledge could be associated with the domain-independent (general) knowledge concerning the maintenance and observation of a solution generating process. The central process of creativity appears to be an ill-structured problem-solving 
process (Simon, 1973). In summary, within creativity studies, a universally agreed classification of knowledge is formulated, which distinguishes between domain-specific knowledge (expertise) and domain-general knowledge (creative-relevant process) (Figure 1).

\subsubsection{Tacit knowledge and creativity}

The concept of tacit knowledge was introduced by Polanyi in 1958. The assertion of knowledge is that, "we can know more than we can tell" (Polanyi, 2009, p. 24). It was also suggested that a "human's intellectual superiority over animals is due to our linguistic capabilities..." (ibid, p. 55) and the information beyond language that is difficult to transfer to others is termed tacit knowledge. In essence it refers to human experiences developed naturally and accumulated over time, and which gradually merges into human life without an explicit explanation. Therefore, tacit knowledge is acquired and developed with engagement and involvement in various activities, including work, play and general life experience. The process of acquiring tacit knowledge is natural and most of the time without the individual being conscious of it, and is typically not possible to be articulated or communicated explicitly. Recently, tacit knowledge has been emphasised by more and more creativity researchers (see Matthew and Sternberg, 2009).

Amabile's (1996) CMC model doesn't include tacit knowledge, but another important aspect of 'intrinsic task motivation', which is deemed equally important as domain knowledge. It is stated that a high level of intrinsic motivation results in a greater probability of demonstrating creativity. So a task being attractive and interesting enough to evoke motivation for engaging in the task is alone considered a crucial factor for creativity. The role of motivation is supported by several other theories that indicate motivation is largely related to and affected by tacit knowledge (Sternberg and Lubart, 1996).

\subsection{Creativity-relevant factors and measurements}

Creativity is "a multi-faceted phenomenon rather than a single unitary construct capable of precise definition" (Rhodes, 1987, p. 218), but which refers to attributes or characteristics of a creative product, process, person/personality, and/or place, namely, the 4Ps (Kozbelt et al., 2010; Gero et al., 2013). Therefore, it is impractical to provide a 'one size fits all' characterisation (Christiaans and Venselaar, 2005). A review of creativity literature in the design domain shows that the majority of studies are focused on a single facet of creativity (Gero et al., 2013). Most of widespread of measurements used are derived from aspects of 4Ps (Cropley, 2000), which implies a close relationship between creativity definitions and its measurements. That is, the instrument for measuring creativity is selected according to how researchers define creativity in a specific study.

In the context of this study we adopted the stance that personal traits are time-invariant, but will partly contribute to the understanding of creativity in the individual (Christiaans, 1992). In addition, we understand that the creativity embedded in a final product outcome may not truly reflect an individual's creativity, as the proportion of creative thinking skill that can be attributed to the individual directly involved (the designer) or other external influences, is difficult to ascertain in any objective manner. This is especially acute in the context of the Chinese design education system where it is acknowledged that tutors often and extensively influence the students in their decision making. Moreover, Gero et al., (2013) state that creativity aspects of personality or place, effect creative outcomes and generally require the mediation of design cognition, rather than perceived impact of creativity through a designed product. As creativity in this study is considered a creative process driven by creative thinking, a combination of divergent and convergent thinking (Cropley, 2000) is central to the process.

\subsection{Literature gap and research hypotheses}

The current literature indicates domain knowledge and tacit knowledge are related to creativity, and the main argument focuses on which category of knowledge would be more important to contribute to creativity (Amabile, 1996; Sternberg and Lubart, 1996). Christiaans and Venselaar (2005) conclude that little is known about the way in which knowledge and skills within or across domains are actually used, and neither the situation of tacit knowledge is applicable, since it remains unclear what kinds of experiences are involved in a certain process (Matthew and Sternberg, 2009). As all categories of knowledge are proposed to be related to creativity, this study intended to examine how each knowledge within these categories is influenced by creativity-relevant factors. 
Metacognition (Figure 2) stands out as a creativity-relevant factor on knowledge application through empirical data collection. The reason is considered twofold;

1. The process of knowledge application is a cognitive process, and individuals usually apply their knowledge without awareness (Gurteen, 1998). It is proposed that cognitive activities may exert impact on such a creative process. From this viewpoint, relevant factors from the view of creative process would be primary to be considered and then examined. Metacognition is then taken into account in this study, which, as Flavell (1979) described

"can lead you to select, evaluate, revise, and abandon cognitive tasks, goals, and strategies in light of their relationships, with one another and with your own abilities and interests with respect to that enterprise". (Flavell, 1979, p. 908).

2. According to the updated literature, the focus of creative process has been shifted from divergent thinking to the interactions (mechanics) between divergent thinking and convergent thinking. The most representative research includes those studies focusing on metacognition, which is considered to be an appropriate criterion for measuring creativity (Puryear, 2015) for its strong relation to creative thinking (Runco, 2006; Kaufman and Beghetto, 2013; Puryear, 2014).

Therefore, based on the three identified categories of knowledge, this research then proposed the following hypotheses that metacognition positively influences the frequencies of applying all three categories of knowledge in FYDP processes (Figure 2).

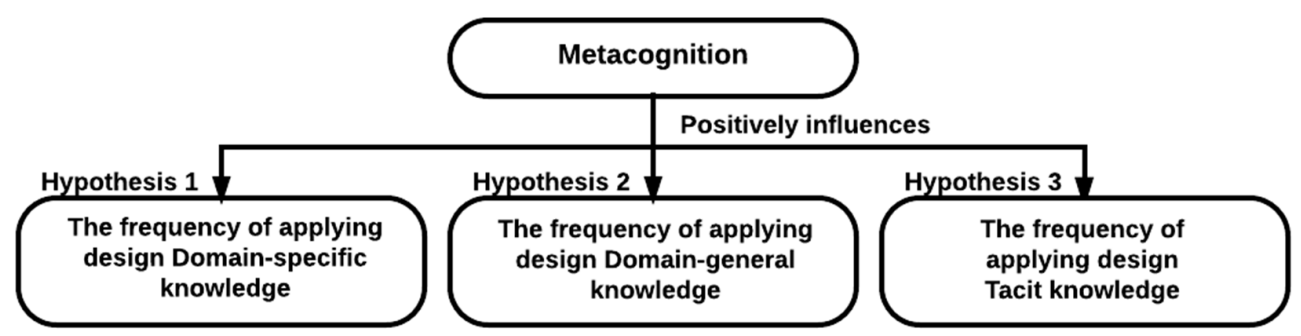

Figure 2. Research hypotheses: Metacognition impacts on knowledge application

\section{Methodology}

\subsection{Investigating knowledge application}

\subsubsection{Developing the knowledge categories}

The literature review identified three categories of knowledge (Domain-specific, Domain-general, and Tacit knowledge). These were subsequently used as a framework for developing a list of knowledge subject areas, known to be expected and applied in FYDPs. In product design research, relevant studies about design knowledge provided an important resource for producing the knowledge subject list for this study. Christiaans and Venselaar (2005) have identified ten items of domain-specific knowledge and one item of domain-general knowledge within industrial/product design. In addition, Popovic's (2004) study referred to strategic knowledge as domain-general knowledge and mentioned the experiential knowledge.

Product/Industrial Design taught programme contents were also surveyed as a means to enrich the knowledge list, as the curriculum often reflects the learning from the imparted knowledge. Three skills were added to the domain-specific knowledge category, and one was added to the domain-general category, sourced from product design course curricula in China (HELPRC, 2015) and the UK QAA report (2016).

Finally, this study introduces tacit knowledge as a specific category of knowledge. There is no relevant reference or validation of related items of this category except acknowledging its strong relationship with personal experiences. We identified three items of general experiences based on Polanyi's (2009) initial ideas of working and daily life experience.

In total, 19 items of knowledge were assembled as the basis of the questions on knowledge application (Table 1). 
Table 1. Developed knowledge list in product design

\section{Domain-specific knowledge:}

K1. Design History: knowledge relates to stylish perspectives

K2. Material: knowledge relates to specific materials to attain certain concept solutions

K3. Design methods: knowledge relates to the application of design research, and design case studies

K4. Aesthetics: knowledge relates to colour, structure and form

K5. Design representation: skills relates to 2D/3D drawing (effect drawing, three views)

K6. User Trials: knowledge relates to simulations of product usage in which subjects are asked to fulfil specified tasks using a product or product simulation.

K7. Client needs: knowledge relates to analysing the design brief

K8. Mechanics

K9. Ergonomics

K10. Skill to operate relevant machines

K11. Media technologies

K12. Knowledge of organization and marketing

K13. Psychology regarding with consumer and user

Domain-general/independent knowledge:

K14. Knowledge of information processing: information searching and analysing

K15. Ill-structured problem-solving process: knowledge relates to analysing situations, defining problems, finding or generating solutions.

K16. Strategies: knowledge relates to motivation, plan and goals.

Tacit Knowledge:

K17. Knowledge of existing design solutions: the precedents of similar project learned

K18. Personal placement experience in design companies

K19. Other experience in daily lives: travelling, reading, events, etc.

Note: K1 - K10, K15 are adopted from Christiaans and Venselaar's (2005) study;

K11-K14 are adopted from HELPRC (2015), China, and QAA report (2016), UK;

K16 is from Popovic's (2004) study;

K17 - K19 are based on Polanyi's (2009) initial ideas on human's experience, and K17, K18 are adopted from HELPRC (2015), China, and the QAA report (2016), UK.

\subsubsection{The psychometric approach}

Clearly there is a challenge in collecting relevant information about the modus operandi of students' knowledge application during FYDPs. The cognitive process of knowledge acquisition occurring without consciousness or realisation by the individual, as it occurs, and the limitation of direct observation or measurement is problematic (Baartman and De Bruijn, 2011).

Hayes (2000) suggested an instrument of psychometric approach, which can be used to investigate cognitive process in the form of a questionnaire to collect data. The primary advantage of this method is to provide the participants with relevant cues for retrospection on a certain process experienced by them. This ensures that the data collected is focused on a specific topic. Moreover, pre-set answers for selecting (usually in numerical, ordinal, or categorical) make the data collection easier for coding and more suitable for statistical analysis. This approach is efficient and cost effective and presents is feasible for a large sample size analysis (Hayes, 2000); whereas the limitation is that it cannot reveal qualitatively deep information concerning the rationales of cognitive activities (Buckingham and Saunders, 2004).

This study adopts this method and constitutes the first attempt in examining the effects of creativityrelevant factor 'metacognition' on knowledge application, because it aims to provide insight into whether it influences knowledge application and how this process is represented, rather than interpreting the reason as to why this process occurs and under what conditions.

\subsubsection{Scaling}

Qualitative responses regarding respondents' feelings, perception, interests, and preferences were measured by Likert scaling (Michell, 1997). One strength of the Likert scale is that it typically provides a large range of responses, which are considered to be discriminating and reliable (Michell, 1997). This study intends to investigate how students apply their knowledge, which focuses on the extent to which 
they apply their individual knowledge during a specific learning process. Thus the 'frequency' of applying knowledge was assessed by asking participants to respond on a 7-point Likert scale from 'never used' to 'always used'.

Based on the developed knowledge list, the 7-point Likert Scale was used to assess the extent to which the students apply each item of knowledge included in the knowledge list. Finally, the 19 questions based on the 19 knowledge items were used to construct an instrument, called 'Self-reported Psychometric Test: How do you apply your knowledge?' in this study.

\subsection{Evaluating creativity abilities by measuring a specific creativity relevant factor - metacognition}

Lai (2011) has provided a review of general methods of metacognition measurement along with the main limitation of each method. For example, self-report and think-aloud techniques allow researchers to access aspects of thinking that are not directly observable but may be likely to underestimate the metacognitive abilities of young children. The approach of teacher observation is somewhat independent of the student's verbal ability and working memory capacity. However, subjectivities may arouse as different teachers would deduce different judgements on the same student. Moreover, most of the existing instruments for measuring metacognition focus on only one or a few aspects of metacognition, such as focusing on metamemory (Schneider, 2008).

This study employed the Metacognitive Awareness Inventory (MAI), developed by Schraw and Dennison (1994), as the measurement of metacognition. The main advantages of MAI in comparison with listed methods are: it is suitable for a large size sample, and is inclusive enough. It is a self-reported measure with 52 items in true-false format, and measures students' metacognition abilities from two aspects 'cognitive knowledge' and 'cognitive regulation'. Moreover, there are several sub-types of cognitive knowledge (declarative, procedural, conditional) as well as that of cognitive regulations (planning, information management, monitoring, debugging, and evaluating) (Schraw and Dennison, 1994), shown in Table 2. Correspondingly, a literature review reveals a consensus on its efficiency as a measurement and this underpins the recommendation of MAI (Akturk and Sahin, 2011).

Table 2. Metacognitive Awareness Inventory (MAI) instrument information

\begin{tabular}{|lcl|}
\hline \multicolumn{1}{c}{ Items } & \\
\hline Overall items & 52 & \\
Cognitive Knowledge & $\mathbf{1 7}$ & \\
Declarative Knowledge & 8 & Knowledge about one's skills, intellectual resources, and abilities \\
Procedural Knowledge & 4 & Knowledge about how to implement learning Procedures/strategies \\
Conditional Knowledge & 5 & Knowledge about when and why to use learning procedures \\
Cognitive Regulations & $\mathbf{3 5}$ & \\
Planning & 7 & Planning, goal setting, and allocating resources prior to learning \\
Information Management & 10 & Skills and strategy sequences used on-line to process information efficiently \\
Monitoring & 7 & Assessment of one's learning or strategy use \\
Debugging & 5 & Strategies used to correct comprehension and performance errors \\
Evaluation & 6 & Analysis of performance and strategy effectiveness after a learning episode \\
\hline Note: Definitions from Schraw and Dennison (1994) \\
\hline
\end{tabular}

\subsection{Questionnaire design and the survey}

The questionnaire consisted of three parts: 1) participants' basic information; 2) Self-reported Psychometric Test: How do you apply your knowledge? 3) Metacognitive Awareness Inventory (MAI). The second and third parts of this questionnaire were two self-assessed instruments intended to examine the different aspects of design students' cognitive behaviours. The students had no need to make any judgement on their cognitive behaviours. They were asked to reflect on their reactions and select the most appropriate answer. For example, to investigate students' knowledge application, the participants were not asked how well they applied knowledge but "how frequently" they apply that knowledge. The MAI survey results might be biased due to over-optimism, but wouldn't necessarily lead to overreaction 
to the "knowledge application" survey. There is a necessary assumption here that participants are neutral and objective as there is no consensus on which cognitive bias is popular amongst the participants.

The tool of the formal online survey was employed to collect data from the final year Product Design students from the UK and China, who had just completed their FYDPs. The total number of valid responses was 375 , with a distribution pattern of 147 from the UK and 228 from China. The time duration of data collection was 51 days from 19-05-2017 to 08-07-2017. The UK data was sourced from the participants with Product Design background, who had attended the "2017 New Designers Exhibition". This event is an independent exhibition held annually in London, and it is the UK's most important graduate design exhibition (https://www.newdesigners.com/). One hundred and forty-seven responded to the on-line questionnaire representing twenty-three of the twenty-eight colleges attending. The data collected from Chinese students was from the top 10 design colleges over a month period by providing target participants with the website link of the survey via email.

\section{Findings and discussions}

\subsection{Main findings}

The research hypotheses assume a positive linear relationship between metacognition and the frequency of applying each type of knowledge. Moreover, the metacognition is the explanatory variable in this relationship. Ordinal Logistical Regression (OLR) (Equation 1) was used to examine these assumptions, which is specifically suitable for those variables measured by the Likert scale.

$$
\text { Yij }=\alpha+\beta_{1} X j+\beta_{2} j \sum X j+\epsilon
$$

Here the dependent variable measures how frequently a student ' $i$ ' applies a specific knowledge item ' $j$ ' on a 7-point Likert scale. 'Yij' is the logarithm of odds of the dependant variable. 'Xj' is the MAI score of participant ' $\mathrm{j}$ '. The ordinal regression enables us to determine how a single unit increase or decrease in MAI is associated with the probability of the dependent variable presenting a higher or lower value. ' $\beta \mathrm{j} \sum \mathrm{Xj}_{\mathrm{j}}$ is the 'sum' of other control factors (gender, age, projects and institutes) which are also examined in the regression to reveal their statistically significant effect on the knowledge application, if any. These control variables are not the research focus of this paper, and are included to be controlled for the potentially significant effect (if any) they may have.

The results are shown in Table 3 below, which report how the application frequency of each knowledge item is influenced by metacognition (note: ${ }^{* * *} \mathrm{p}<0.01,{ }^{*} \mathrm{p}<0.05,{ }^{*} \mathrm{p}<0.1$ ), and they are summarised in three categories:

1. The metacognition's impact on the applications of 8 knowledge items (marked in Bold) are statistically significant and positive ( $p$-value $<0.1$, 'Coefficient' $>0$ ) in both the students from UK and China. Amongst them, 4 items belong to the domain-specific knowledge; 1 item is from domain-general knowledge, and 3 items are from tacit knowledge.

2. The metacognition's impact on the applications of 6 knowledge items (marked in Italic) are statistically insignificant (p-value $>0.1$ ) in both the students from UK and China. Amongst them, 5 items are from domain-specific knowledge, and 1 item is from domain-general knowledge.

3. There are 5 items (4 from domain-specific knowledge, 1 item from domain-general knowledge) showing different results between the UK data and China data.

For these knowledge items as identified in category 1, whether they are more likely to be more frequently or less frequently used (on a 7-point Likert scale), is largely determined/explained by whether the corresponding participants are of higher or lower MAI. This implies that the higher is the MAI of the participants, the higher are their chances of more frequent application of the category one knowledge items. This, in turn, indicates that the category 1 knowledge items, via how frequently they are used, are related to students' creativity. Metacognition in this study is a criterion for assessing creative thinking ability, and the students who got higher score of MAI test are considered to have advantage in creative thinking, and thus being more creative. For the category 2 knowledge items, how frequently they are used, is not influenced by the MAI levels of the participants. For the category 3 knowledge items, the evidence found in the students of UK and China are contradictory, thus implying that the cultural 
differences may constitute another underlying factor for knowledge application. As the application of knowledge items in category 2 are not influenced by metacognition, the subsequent discussions is focused specifically on knowledge items in category 1 and 3.

Table 3. Regression of how metacognition influences knowledge application

\begin{tabular}{|c|c|c|c|c|c|c|}
\hline \multirow[t]{2}{*}{ MAI } & \multicolumn{2}{|c|}{ Coefficient } & \multicolumn{2}{|c|}{$z$-value } & \multicolumn{2}{|r|}{ p-value } \\
\hline & UK & China & UK & China & UK & China \\
\hline K1 & .0124154 & .0178451 & 0.50 & 0.93 & 0.616 & 0.354 \\
\hline K2 & .0880546 & .0390951 & 3.18 & 1.90 & $0.001 * * *$ & $0.058 *$ \\
\hline K3 & -.0227451 & .0042632 & -0.92 & 0.22 & 0.358 & 0.829 \\
\hline K4 & .0048117 & .0418545 & 0.18 & 2.11 & 0.853 & $0.034 * *$ \\
\hline K5 & .0071817 & -.0095757 & 0.29 & -0.48 & 0.774 & 0.633 \\
\hline K6 & .0707227 & .042094 & 2.71 & 2.08 & $0.007 * * *$ & $0.037 * *$ \\
\hline K7 & . 0790986 & . 0135135 & 3.03 & 0.72 & $0.002 * * *$ & 0.474 \\
\hline K8 & .0679747 & .048247 & 2.52 & 2.22 & $0.012 * *$ & $0.026 * *$ \\
\hline K9 & .0836136 & .0570645 & 3.11 & 2.84 & $0.002 * * *$ & $0.004 * * *$ \\
\hline K10 & -.0098781 & .0465495 & -0.40 & 2.29 & 0.691 & $0.022 * *$ \\
\hline K11 & -.0342168 & .0129069 & -1.37 & 0.64 & 0.171 & 0.523 \\
\hline K12 & .0423462 & .0620578 & 1.63 & 3.01 & 0.103 & $0.003 * * *$ \\
\hline K13 & .0355244 & .0102593 & 1.39 & 0.51 & 0.164 & 0.609 \\
\hline K14 & . 1101319 & -.0049528 & 4.06 & -0.25 & $0.000 * * *$ & 0.803 \\
\hline K15 & .0287052 & .0280019 & 1.12 & 1.39 & 0.264 & 0.165 \\
\hline K16 & .0960231 & .040478 & 3.44 & 1.98 & $0.001 * * *$ & $0.048 * *$ \\
\hline K17 & .1129527 & .0671738 & 3.92 & 3.07 & $0.000 * * *$ & $0.002 * * *$ \\
\hline K18 & .0984483 & .0670912 & 3.71 & 3.31 & $0.000 * * *$ & $0.001 * * * *$ \\
\hline K19 & .1019051 & .0797108 & 3.59 & 3.74 & $0.000^{* * * *}$ & $0.000^{* * *}$ \\
\hline
\end{tabular}

\subsection{Discussion}

\subsubsection{Knowledge items in category 1}

The eight items in category one are provided in Table 4.

Table 4. Knowledge items whose applications are influenced by metacognition

K2. Material: knowledge relates to specific materials to attain certain concept solutions
K6. User Trials: knowledge relates to simulations of product usage in which subjects are asked to
fulfil specified tasks using a product or product simulation
K8. Mechanics
K9. Ergonomics
K16. Strategies: knowledge relates to motivation, plan and goals
K17. Knowledge of existing design solutions: the precedents of similar project you have learned
K18. Personal placement experience in design companies
K19. Other experience in daily lives (for example travel, reading, etc.)

$K 2$, K6, K8, and $K 9$ : These knowledge items belong to domain-specific knowledge, and they evidence to have something in common in the design process. Although the outcomes of applying them seem directly to be reflected in the designed product (the surface, the structure, the function and the performance), they are all suggested to be considered at the very beginning of the design process (Sagot et al., 2003; Ljungberg and Edwards, 2003; Brown, 2009; Bingham et al., 2013). Each knowledge aims to address and solve a specific design problem. The solution is developed and refined gradually during the design process, and eventually, the solution of this problem evolves and matures to be physically materialised in the final product. Therefore, the domain-specific knowledge $\mathrm{K} 2, \mathrm{~K} 6, \mathrm{~K} 8$, and $\mathrm{K} 9$ play the significant roles in the subject of the problem space transference to solution space. In other words, the designer's conceptual ideas 
become more and more clear and are manifestly delivered and embodied as a physical product. According to Acuna and Sosa (2010), the process of transferring conceptual ideas into the form of physical object fertilise the creativity. This, in turn, may explain why these participants with higher scores of MAI test are considered to be more creative, are more likely to apply this kind of knowledge at a higher frequency in the FYDP process. It may be that their better creative thinking abilities help them better understand the effects of applying this knowledge, which is highly integrated throughout the whole design process rather than at a specific stage of the design process. The design process is solution-oriented and correspondingly this knowledge is applied throughout, which in turn helps to facilitate a smoother FYDP engagement process. In contrast, those students with relatively lower scores of MAI test are considered to be less creative, are more likely to apply this knowledge at a lower frequency. This could probably be attributed to their increased attention on specific stages when using this knowledge (this may be influenced by the course module), which indicates they are relatively weak on using this knowledge at the appropriate stages. Creativity in design is known as problem-based and solution-focused and has been studied mainly via qualitative approach and based on practices (Friedman, 2003). The results here, go some way to support this statement from a quantitative perspective by identifying the relationship between creative abilities of individuals via their metacognition and the frequency of a few certain design knowledge applications.

Concluding, not all items (only K2, K6, K8, K9) in domain-specific knowledge are found to be significantly influenced by metacognition as assumed in Hypothesis 1, therefore it is suggested that the Hypothesis 1 is supported by these results from K2, K6, K8, K9.

K16: This knowledge item belongs to domain-general knowledge. The results show that frequency of $\mathrm{K} 16$ application (domain-general knowledge) is positively influenced by students' metacognition. On the one hand, strategy knowledge should be involved in the design process at the early stage, such as during the establishment of a range of goals and plans. On the other hand, it also manages to deploy different knowledge scopes and use them effectively along the design process (Alexander and Judy, 1988). Similarly, Christiaans and Venselaar's (2005) stated the domain-general knowledge usually guides the application of domain-specific knowledge. As implied by the study conducted by Christiaans and Venselaar (2005), students whose designs have a higher creativity rating would on average elicit a greater amount of domain-general knowledge than other students, which plays a crucial role in the design process as a 'guideline'. It is implied here that students with higher MAI score intend to use this knowledge at a high frequency. Whereas those students with lower MAI score would probably ignore it's 'guideline' role in the whole process, and hence present a relatively lower frequency of this knowledge application. The 'strategy' knowledge tends to influence the whole design process, and thus may also influence the application of some domain-specific knowledge in the process. Those students with lower MAI use K16 less frequently, indicating their limitation in a proper management of the whole process. Hence they may apply certain domain-specific knowledge less efficiently, and may face more problems in the process, as the research problem states.

Not all items in domain-general knowledge are found to be significantly influenced by metacognition as assumed in Hypothesis 2. Hypothesis 2 is supported by the result from K16.

K17, K18, and K19: These knowledge items are related to tacit knowledge. The study conducted by Dorst and Cross (2001) indicates that the design process is a 'co-evolution' process varying with the problem space and the solution space, rather than a technical ill-structured problem-solving process. Moreover, Cross (2004) states that the designers' perceptual activities underlying creative insight is not so much a 'leap', but more akin to 'bridging' between problem space and solution space. This 'bridging' would be achieved depending on the magnitude of designer's reflective activities (Dorst and Cross, 2001). The tacit knowledge is, thus considered to be a significant factor, possibly because it is one of the key materials to facilitate the process of reflection in such a 'co-evolution' and 'bridging' process (Cross, 2004). Kolb (1984) stated that to obtain this so-called experience-based knowledge, reflection on experience has thus been advocated as a primary component in management and education literature. It is therefore, possibly unsurprising that creative students would like to apply tacit knowledge more frequently, as they are good at connecting current situation with their experiences.

The results indicate that applications of all three items in tacit knowledge are significantly influenced by metacognition, supporting Hypothesis 3 . 


\subsubsection{Knowledge items in category 3}

The five items in category three are provided in Table 5.

Table 5. Knowledge items whose application influenced by metacognition shows different results between the UK data and China data

\begin{tabular}{l}
\hline P-values indicate the influence is significant in China, whereas insignificant in the UK: \\
K4. Aesthetics: knowledge relates to colour, structure and form. \\
K10. Skill to operate relevant machines \\
K12. Knowledge of organisation and marketing \\
\hline P-values indicate the influence is significant in the UK, whereas insignificant in China: \\
K7. Client needs: knowledge regarding to analyse the design brief \\
K14. Information processing
\end{tabular}

The students with relatively higher scores of MAI in both countries tend to apply knowledge in different ways, influenced by their respective understandings of the market and the current trend of technology due to the social and cultural differences. These results further support the socio-culture viewpoint in the study of creativity from a new perspective. The Chinese evaluate creativity from the viewpoint of "novelty" (Lan and Kaufman, 2012) and perceive creativity as an "intuitive" and "incremental" process during which all social elements continually accumulate (e.g. civilisation) (Paletz and Peng, 2008). This may explain why Chinese creative students are more likely to apply K4 and K10, as both knowledge items largely reflect the "Art and Craft" attributes of design, which are specifically emphasised in China (Wu, 2001) and usually deemed to inspire novelty and reflects traditional understandings of design (e.g. the forms and the crafts of artificial) (Kuma, 2008). On the other hand, the westerners evaluate creativity from the view of "appropriateness", so more attention is given to the rationale of the process (Paletz and Peng, 2008), and emphasise the importance of new "information seeking" (K14). Therefore, they tend to accept a more "radical" perspective of creativity. Furthermore, the UK creative students prefer to apply more knowledge of "client's needs" (K7), on the contrary, the Chinese creative students prefer to apply more knowledge of "organisation and mass marketing" (K12) probably due to different design methods being taught in each respective country. In the UK Design Higher Education, the emphasis is on the 'user centred design' processes (Zoltowski et al., 2012) whereas in China's Design Higher Education the focus is on the 'form and function' (Buchanan, 2004, p. 30).

\subsection{Implications}

The outcomes suggest that we may be able to bring more practical and directional guidance in product design education, which can be summarised as:

1. Determining the kinds of knowledge to be emphasised in the curriculum as applying them may be more positive to lead creative process, for example, those knowledge items in category 1 (Table 4).

2. Design tutors could encourage student reflection on experiences when certain stage of an FYDP process stalls.

3. Design tutors may cultivate students' awareness on culture-related knowledge, such as those items in category 3 (Table 5), which could facilitate the communication in a co-operate design process (e.g. involving students from different cultures).

\section{Conclusion}

This study investigated the relationship between a creativity relevant factor (metacognition) and knowledge application within the context of product design education. The final year design project (FYDP) process was used to explore the utilisation of student knowledge. The study reveals insights that suggest a new way of categorising knowledge according to the influences excerpted by metacognition, which may inform design education practices. Specifically, it may extend academic support in FYDPs for both the design students and educators. In addition, there may be a benefit from developing an understanding of which knowledge type should be encouraged when engaging in certain project aspects. As a first step this study provides some insights into how metacognition impacts other 
cognitive processes (i.e. knowledge application) in design education. Initial results illustrate the importance of exploring knowledge application in future research, to provide insight into improved practices within and across educational and creative domains.

\section{References}

Acuna, A. and Sosa, R. (2010), “The Complementary Role of Representations in Design Creativity: Sketches and Models", In: Taura, T. and Nagai, Y. (Eds.), Design Creativity 2010, Springer, London, pp. 265-270. https://doi.org/10.1007/978-0-85729-224-7 34

Adams, K. (2005), The Sources of Innovation and Creativity, National Centre on Education and the Economy.

Akturk, A.O. and Sahin, I. (2011), "Literature review on metacognition and its measurement", Procedia-Social and Behavioral Sciences, Vol. 15, pp. 3731-3736. https://doi.org/10.1016/j.sbspro.2011.04.364

Alexander, P.A. and Judy, J.E. (1988), "The interaction of domain-specific and strategic knowledge in academic performance", Review of Educational research, Vol. 58 No. 4, pp. 375-404.

Alexander, P.A., Schallert, D.L. and Hare, V.C. (1991), "Coming to terms: How researchers in learning and literacy talk about knowledge", Reviewer Educational Research, Vol. 61 No. 3, pp. 315-345. https://doi.org/10.3102/00346543061003315

Amabile, T.M. (1996), Creativity in context: Update to "the social psychology of creativity, Westview press.

Baartman, L.K. and De Bruijn, E. (2011), "Integrating knowledge, skills and attitudes: Conceptualising learning processes towards vocational competence", Educational Research Review, Vol. 6 No. 2, pp. 125-134. https://doi.org/10.1016/j.edurev.2011.03.001

Bingham, G., Southee, D. and Page, T. (2013), "An integrated approach for the teaching of mechanics and electronics in a design context", Proceedings of E\&PDE 2013, the 15th International Conference on Engineering and Product Design Education, Dublin, Ireland, September 5-6, 2013, pp. 170-175.

Brown, T. (2009), Change by Design: How Design Thinking Creates New Alternatives for Business and Society: How Design Thinking Can Transform Organizations and Inspire Innovation, HarperCollins, New York, NY.

Buchanan, R. (2004), "Human-centered Design: Changing Perspectives on Design Education in the East and West”, Design Issues, Vol. 20 No. 1, pp. 30-39. https://doi.org/10.1162/074793604772933748

Buckingham, A. and Saunders, P. (2004), The survey methods workbook: From design to analysis, Polity Pr.

Christiaans, H. and Venselaar, K. (2005), "Creativity in design engineering and the role of knowledge: Modelling the expert", International Journal of Technology and Design Education, Vol. 15 No. 3, pp. 217-236. https://doi.org/10.1007/s10798-004-1904-4

Christiaans, H.H.C.M. (1992), Creativity in design: the role of domain knowledge in designing, Uitgeverij Lemma, Utrecht.

Cropley, A. (2006), "In praise of convergent thinking”, Creativity Research Journal, Vol. 18 No. 3, pp. 391-404. https://doi.org/10.1207/s15326934crj1803_13

Cropley, A.J. (2000), "Defining and measuring creativity: Are creativity tests worth using?", Roeper Review, Vol. 23 No. 2, pp. 72-79.

Cross, N. (2004), "Expertise in design: an overview", Design Studies, Vol. 25 No. 5, pp. 427-441. https://doi.org/10.1016/j.destud.2004.06.001

Dorst, K. and Cross, N. (2001), "Creativity in the design process: co-evolution of problem solution", Design studies, Vol. 22 No. 5, pp. 425-437. https://doi.org/10.1016/S0142-694X(01)00009-6

Flavell, J.H. (1979), "Metacognition and cognitive monitoring: A new area of cognitive-developmental theory", American Psychologist, Vol. 34 No. 10, pp. 906-911.

Friedman, K. (2003), "Theory construction in design research: criteria: approaches, and methods", Design Studies, Vol. 24 No. 6, pp. 507-522. https://doi.org/10.1016/S0142-694X(03)00039-5

Gero, J.S., Jiang, H. and Williams, C.B. (2013), "Design cognition differences when using unstructured, partially structured, and structured concept generation creativity techniques", International Journal of Design Creativity and Innovation, Vol. 1 No. 4, pp. 196-214. https://doi.org/10.1080/21650349.2013.801760

Gurteen, D. (1998), "Knowledge, creativity and innovation”, Journal of Knowledge Management, Vol. 2 No. 1, pp. 5-13. https://doi.org/10.1108/13673279810800744

Hayes, N. (2000), Doing psychological research, Taylor \& Francis Group.

HELPRC (2015), Evaluation of Undergraduate Teaching and Learning in Higher Education (Section of Art and Design), Higher Education Law of the People's Republic of China (2015 Amendment).

Kaufman, J.C. and Beghetto, R.A. (2013), "In Praise of Clark Kent: Creative Metacognition and the Importance of Teaching Kids When (Not) to Be Creative", Roeper Review, Vol. 35 No. 3, pp. 155-165. https://doi.org/10.1080/02783193.2013.799413

Kolb, D.A. (1984), Experiential learning, Englewood Cliffs. 
Kozbelt, A., Beghetto, R.A. and Runco, M.A. (2010), “Theories of Creativity”, In: Kaufman, J.C. and Sternberg, R.J. (Eds.), Cambridge Handbook of Creativity, Cambridge University Press, New York, pp. $20-47$. https://doi.org/10.1017/cbo9780511763205.004

Kuma, K. (2008), Anti-object: the dissolution and disintegration of architecture (Vol. 2), AA Publishing.

Lai, E.R. (2011), Metacognition: A literature review. [online] Available at: http://com/images/tmrs/Metacognition_Literature Review_Final.pdf on (accessed 17.07.2015).

Lan, L. and Kaufman, J.C. (2012), "American and Chinese similarities and differences in defining and valuing creative products", The Journal of Creative Behavior, Vol. 46 No. 4, pp. 285-306. https://doi.org/10.1002/jocb.19

Lawson, B. (2006), How designers think: The design process demystified, Routledge.

Ljungberg, L.Y. and Edwards, K.L. (2003), "Design, materials selection and marketing of successful products", Materials \& Design, Vol. 24 No. 7, pp. 519-529. https://doi.org/10.1016/S0261-3069(03)00094-3

Matthew, C.T. and Sternberg, R.J. (2009), "Developing experience-based (tacit) knowledge through reflection", Learning and Individual Differences, Vol. 19 No. 4, pp. 530-540. https://doi.org/10.1016/j.lindif.2009.07.001

Michell, J. (1997), "Quantitative science and the definition of measurement in psychology", British Journal of Psychology, Vol. 88 No. 3, pp. 355-383. https://doi.org/10.1111/j.2044-8295.1997.tb02641.x

Paletz, S. and Peng, K. (2008), "Implicit theories of creativity across cultures: Novelty and appropriateness in two product domains", Journal of Cross Cultural Psychology, Vol. 39 No. 3, pp. $286-302$. https://doi.org/10.1177/0022022108315112

Polanyi, M. (2009), The tacit dimension, University of Chicago press.

Popovic, V. (2004), "Expertise development in product design—strategic and domain specific knowledge connections", Design Studies, Vol. 25 No. 5, pp. 527-545. https://doi.org/10.1016/j.destud.2004.05.006

Puryear, J.S. (2014), "Inside the creative sifter: Recognizing metacognition in creativity development", The Journal of Creative Behavior, Vol. 50 No. 4, pp. 321-332. https://doi.org/10.1002/jocb.80

Puryear, J.S. (2015), "Metacognition as a Moderator of Creative Ideation and Creative Production", Creativity Research Journal, Vol. 27 No. 4, pp. 334-341. https://doi.org/10.1080/10400419.2015.1087270

QAA (2016), Subject Benchmark Statement Art and Design: Draft for consultation, QAA.

Rhodes, M. (1987), “An analysis of creativity”, In: Isaksen, S.G. (Ed.), Frontiers of creativity research: Beyond the basics, Bearly, Buffalo, NY, pp. 216-222.

Runco, M.A. (2006), "Introduction to the special issue: Divergent thinking”, Creativity Research Journal, Vol. 18 No. 3, pp. 249-250. https://doi.org/10.1207/s15326934crj1803_1

Sagot, J.C., Gouin, V. and Gomes, S. (2003), "Ergonomics in product design: safety factor", Safety Science, Vol. 41 No. 2, pp. 137-154. https://doi.org/10.1016/S0925-7535(02)00038-3

Schneider, W. (2008), "The development of metacognitive knowledge in children and adolescents: Major trends and implications for education", Mind, Brain, and Education, Vol. 2 No. 3, pp. 114-121. https://doi.org/0.1111/j.1751-228X.2008.0004.x

Schraw, G. and Dennison, R.S. (1994), "Assessing metacognitive awareness", Contemporary Educational Psychology, Vol. 19 No. 4, pp. 460-475. https://doi.org/10.1006/ceps.1994.1033

Simon, H.A. (1973), “Applying information technology to organization design”, Public Administration Review, Vol. 33 No. 3, pp. 268-278. https://doi.org/10.2307/974804

Sternberg, R.J. (2003), "Creative thinking in the classroom", Scandinavian Journal of Educational Research, Vol. 47 No. 3, pp. 325-338. https://doi.org/10.1080/00313830308595

Sternberg, R.J. and Lubart, T.I. (1996), "Investing in creativity", American psychologist, Vol. 51 No. 7, pp. 677688. https://doi.org/10.1037/0003-066X.51.7.677

Weisberg, R.W. (1999), "Creativity and knowledge: A challenge to theories”. In: Sternberg, R.J. (Ed.), Handbook of Creativity, Cambridge University Press, New York, pp. 226-248.

Wu, L. (2001), "Foreign course modules and the unsuccessful "localization": a case study of pattern, sketch and structure", Chinese Art Education, Vol. 6, pp. 15-18.

Zhang, Y., Bohemia, E. and McCardle, J. (2017), "Review of Creativity Factors in Final Year Design Projects in China", Proceedings of the 19th International Conference on Engineering and Product Design Education (E\&PDE17), Building Community: Design Education for a Sustainable Future, The Design Society, pp. 382-387.

Zoltowski, C.B., Oakes, W.C. and Cardella, M.E. (2012), "Students' Ways of Experiencing Human-Centered Design”, Journal of Engineering Education, Vol. 101 No. 1, pp. 28-59. https://doi.org/10.1002/j.21689830.2012.tb00040.x

Yang Zhang, PhD student

Loughborough University, Design School

25 Coniston Crescent, LE11 3RQ Leicester, United Kingdom

Email: y.zhang4@lboro.ac.uk 